\title{
Approaching Process Mining with Sequence Clustering: Experiments and Findings
}

\author{
Diogo Ferreira ${ }^{1,3}$, Marielba Zacarias ${ }^{2,3}$, Miguel Malheiros ${ }^{3}$, Pedro Ferreira $^{3}$ \\ ${ }^{1}$ IST - Technical University of Lisbon, Taguspark, Portugal \\ ${ }^{2}$ Universidade do Algarve, ADEEC-FCT, Faro, Portugal \\ ${ }^{3}$ Organizational Engineering Center, INOV, Lisbon, Portugal \\ diogo.ferreira@ist.utl.pt,mzacaria@ualg.pt, \{miguel.malheiros, pedro.romeu\}@gmail.com
}

\begin{abstract}
Sequence clustering is a technique of bioinformatics that is used to discover the properties of sequences by grouping them into clusters and assigning each sequence to one of those clusters. In business process mining, the goal is also to extract sequence behaviour from an event log but the problem is often simplified by assuming that each event is already known to belong to a given process and process instance. In this paper, we describe two experiments where this information is not available. One is based on a real-world case study of observing a software development team for three weeks. The other is based on simulation and shows that it is possible to recover the original behaviour in a fully automated way. In both experiments, sequence clustering plays a central role.
\end{abstract}

Keywords. Process Mining, Sequence Clustering, Task Identification, Process Discovery, Workflow Logs

\section{Introduction}

In bioinformatics, sequence clustering algorithms have been used to automatically group large protein datasets into different families [12,13], to search for protein sequences that are homologous to a given sequence [17], and to map or align a given DNA sequence to an entire genome [20], to cite only some of the most common applications. In all of these applications, sequence clustering becomes a valuable tool to gain insight into otherwise seemingly senseless sequences of data.

A similar kind of challenge arises in process mining, where the goal is to extract meaningful task sequences from an event log, usually resorting to special-purpose algorithms that can recover the original workflow that produced the log [1].

The idea of applying sequence clustering to process mining comes at a time when process mining is still heavily dependent on the assumption that the event log contains "sufficient" information [4], i.e., that each event in the log is clearly associated with a specific activity and case (process instance) [1]. This comes as a major disadvantage since (1) the classes of information systems that are able to generate such logs are restricted to process-aware systems, and (2) it becomes impossible to apply and benefit from process mining in scenarios where the log data is not available in that form. 
A sequence clustering approach can alleviate these requirements by grouping similar sequences and identifying typical ones without the need to provide any input information about the business logic. Of course, the results will bear a degree of uncertainty, whereas process mining approaches typically aim at finding exact models. Still, sequence clustering can provide valuable insight into the kind of sequences that are being executed.

The paper is structured as follows: section 2 provides an overview of process mining approaches, and section 3 presents the sequence clustering algorithm. Then sections 4 and 5 describe two different experiments and report on the problems encountered and the results obtained.

\section{Process mining approaches}

In general, all process mining approaches take an event log as input and as a starting point for the discovery of underlying processes. The event log (also called process trace or audit trail) is list of records resulting from the execution of some process. For the log to be "minable", each record usually contains information about the activity that was executed, the process instance that it belongs to, and the time of execution. The requirements on the log, i.e. the kind of information it should contain, varies according to the process mining algorithm being used.

In fact, it is the choice of mining algorithms that often leads to different process mining approaches. Some of the algorithms used for process mining include:

- the $\alpha$-algorithm [4] - an algorithm that is able to re-create the Petri-net workflow from the ordering relations found in the even log. For the algorithm to work, the log must contain the process instance identifier (case id) and it must be rather complete in the sense that all ordering relations should be present in the log.

- inference methods [8] - a set of three different algorithms used to infer a finite state machine (FSM) from an event log, where the log is regarded as a simple sequence of symbols. The three algorithms represent different levels of compromise between accuracy and robustness to noise. The MARKOV algorithm, inspired by Markov models, seems to be the most promising. The algorithm works by building up an event graph as the result of considering Markov chains with increasing order. In the last step, the graph is converted to a FSM, which represents the process that was found.

- directed acyclic graphs [5] - an algorithm that is able to generate a dependency graph from a workflow system log. The log must contain a relatively high number of executions of the same process so that the dependency graph for that process can be completely built. Originally, the algorithm was proposed to support the adoption of workflow systems rather than actually pursuing process mining.

- inductive workflow acquisition [16] - an approach in which the goal is to find a hidden markov model (HMM) that best represents the structure of the original process. The HMM can be found by either top-down or bottom-up refinement of an initial HMM structure; these are known as model splitting and model merging 
algorithms, respectively. The initial HMM structure is built directly from the log, which is regarded as a simple sequence of symbols. Reported results suggest that model splitting is faster and more accurate than model merging.

- hierarchical clustering [14] - an algorithm that, given a large set of execution traces of a single process, separates them into clusters and finds the dependency graph separately for each cluster. The clusters of workflow traces are organized into a tree, hence the concept of model hierarchy. After the workflow models for the different clusters have been found, a bottom-up pass through the tree generalizes them into a single one.

- genetic algorithm [2] - an algorithm in which several candidate solutions are evaluated by a fitness function that determines how consistent each solution is with the log. Every solution is represented by a causal matrix, i.e. a map of the input and output dependencies for each activity. Candidate solutions are generated by selection, crossover and mutation as in typical genetic algorithms. The search space is the set of all possible solutions with different combinations of the activities that appear in the event $\log$. The log should contain a relatively high number of execution traces.

- instance graphs [10] - an approach that aims at portraying graphical representations of process execution, especially using Event-driven Process Chains (EPCs). For each execution trace found in the log, an instance graph is obtained for that process instance. In order to identify possible parallelism, each instance graph is constructed using the dependencies found in the entire log. Several instance graphs can then be aggregated in order to obtain the overall model for that $\log$ [11].

In general, as far as input data is concerned, all these algorithms require an event log that contains several, if not a very large number, of execution traces of the same process instance. (An exception is the RNET algorithm used in [8] which can receive a single trace as training input, but the results can vary widely depending on that given input sequence.) Because the log usually contains the traces of multiple instances, it is also required to have labelling field - usually called the case id [1] - which specifies the process instance for every recorded event.

Another requirement on the content of the event $\log$ is that, for algorithms such as [4] and [10], which rely on finding causal relations in the log, task $A$ can be considered the cause of task $B$ only if $B$ follows $A$ but $A$ never follows $B$ in the $\log$. Exceptional behaviour, errors or special conditions that would make $A$ appear after $B$ could ruin the results. These conditions are referred to as noise; algorithms that are able to withstand noise are said to be robust to noise [3]. Most algorithms can become robust to noise by discarding causal relations with probability below a given threshold; this threshold is usually one of the algorithm parameters.

The problem with these requirements is that they may be difficult to apply in many potential scenarios for process mining. For example, in some applications the case id may be unavailable if the log is just an unclassified stream of recorded events. In other applications, it may be useful to clearly identify and distinguish normal behaviour from exceptional one, without ruling out small variations simply as noise. These issues suggest that other kind of algorithms could provide valuable insight into the original behaviour that produced the log. If there is no case id available, and there is an 
unpredictable amount of ad-hoc behaviour, then an algorithm that allows us to sort out and understand that behaviour could be the first step before actually mining those processes. Sequence clustering algorithms are a good candidate for this job.

\section{Sequence clustering}

Sequence clustering is a collection of methods that aim at partitioning a number of sequences into meaningful clusters or groups of similar sequences. The development of such methods has been an active field of research especially in connection with challenges in bioinformatics [7]. Here we will present the basic principles by referring to a simple sequence clustering algorithm based on first-order Markov chains [6].

In this algorithm, each cluster is associated with a first-order Markov chain, where the current state depends only on the previous state. The probability that an observed sequence belongs to a given cluster is in effect the probability that the observed sequence was produced by the Markov chain associated with that cluster. For a sequence $\boldsymbol{x}=\left\{x_{0}, x_{1}, x_{2}, \ldots, x_{L-1}\right\}$ of length $L$ this can be expressed simply as:

$$
p\left(\boldsymbol{x} \mid c_{k}\right)=p\left(x_{0}, c_{k}\right) \cdot \prod_{i=1}^{i=L-1} p\left(x_{i} \mid x_{i-1}, c_{k}\right)
$$

where $p\left(x_{0}, c_{k}\right)$ is the probability of $x_{0}$ occurring as the first state in the Markov chain associated with cluster $c_{k}$ and $p\left(x_{i} \mid x_{i-1}, c_{k}\right)$ is the transition probability of state $x_{i-1}$ to state $x_{i}$ in that same Markov chain. Given the way to compute $p\left(\boldsymbol{x} \mid c_{k}\right)$, the sequence clustering algorithm can be implemented as an extension to the well-known Expectation-Maximization (EM) algorithm [9]. The steps are:

1. Initialize the model parameters $p\left(x_{0}, c_{k}\right)$ and $p\left(x_{i} \mid x_{i-1}, c_{k}\right)$ randomly, i.e. for each cluster the state transition probabilities of the associated Markov chain are initialized at random.

2. Using the current model parameters, assign each sequence to each cluster with a probability given by equation (1).

3. Use the results of step 2 to re-estimate the model parameters, i.e. recalculate the state transition probabilities of each Markov chain based on the sequences that belong to that cluster.

4. Repeat steps 2 and 3 until the mixture model converges.

This sequence clustering algorithm has been implemented in Microsoft SQL Server $2005^{\circledR}[19]$ and is readily available for use either programmatically via an OLE DB for Data Mining interface [18] or via a user-friendly interface in Microsoft Visual Studio $2005^{\circledR}$.

In either case, the algorithm must be provided with two input tables: a case table and a nested table. The case table contains one record for each sequence; it conveys the number of sequences in the input data set together with some descriptive information about each sequence. The nested table contains the steps for all sequences, where each step is numbered and labelled. The number is the order of occurrence within the sequence, and the label is a descriptive attribute that denotes the state in a Markov chain. The case and nested tables share a one-to-many relationship: each sequence in the case table is associated with several steps in the nested table. The connecting 
attribute, which is application-specific, serves as key in the case table and as sequence scope delimiter in the nested table.

For the sake of clarity, let us consider a simple example. Suppose the members of a given family have different ways of zapping through TV channels according to their own interests. Let us assume that each member always finds the TV switched off, and after turning it on, goes through a set of channels before turning it off again. Every time it is turned on, the TV generates a new session identifier (session id) and records both session-related information as well as the sequence of channel changes. Figure 1 shows the case and nested tables for this scenario. The session identifier is both the key to the case table and the sequence scope delimiter for the nested table. The case table contains descriptive, non-sequence attributes about each session, whereas the nested table contains the steps for each sequence, both numbered and labelled.

\begin{tabular}{|l|l|} 
sessionid & timeofday \\
\hline 1234 & $2007-04-0919: 41: 00$ \\
\hline 1235 & $2007-04-0921: 15: 00$ \\
\hline 1236 & $2007-04-1018: 56: 00$ \\
\hline 1237 & $2007-04-1020: 38: 00$ \\
\hline 1238 & $2007-04-1021: 25: 00$ \\
\hline 1239 & $2007-04-1119: 06: 00$ \\
\hline 1240 & $2007-04-1120: 23: 00$ \\
\hline
\end{tabular}

(a)

\begin{tabular}{|l|l|l|} 
sessionid & channel & numchange \\
\hline 1234 & Music & 1 \\
\hline 1234 & Music & 2 \\
\hline 1234 & Movies & 3 \\
\hline 1234 & Movies & 4 \\
\hline 1235 & News & 1 \\
\hline 1235 & Sports & 2 \\
\hline 1235 & News & 3 \\
\hline
\end{tabular}

(b)

Fig. 1. Case (a) and nested (b) tables for the simple TV usage scenario

It can be seen from this simple example that the input data to be provided to the sequence clustering algorithm already has a form of case $i d$, which is the session identifier. Pre-processing techniques will have to be used to assign this case id if it is not available in the first place. We will look at this problem ahead in the context of two different experiments. What is interesting to note here is the kind of results that the sequence clustering algorithm is able to produce. Figure 2 shows four of the clusters that the algorithm was able to identify from a given set of 24 sequences for the simple TV usage scenario. Each cluster has a different Markov chain that is able to generate the sequences assigned to that cluster. This effectively captures the dominant behaviour of similar sequences.

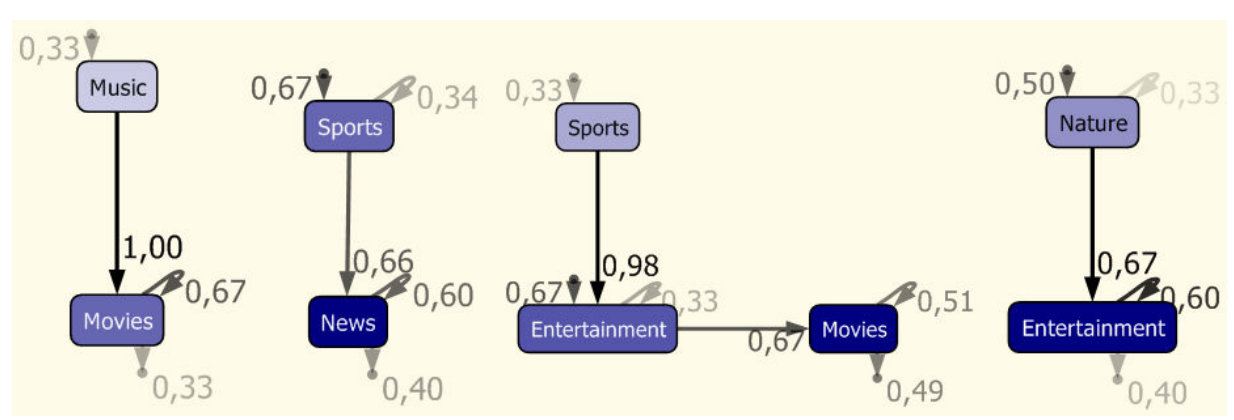

Fig. 2. The Markov chains in four of the clusters obtained for the simple TV usage scenario. 
The number of clusters to be found can be set manually or automatically by letting the algorithm perform a heuristic to determine the number of clusters for the given data. This is usually very useful to use as an initial guess before trying to run the algorithm with different parameters.

To produce the results shown in figure 2 the algorithm performed a number of iterations, where each iteration comprises two steps: the expectation step and the maximization step. In the expectation step the algorithm assigns each sequence $\boldsymbol{x}$ to the cluster $c_{k}$ that gives the highest membership probability $p\left(\boldsymbol{x} \mid c_{k}\right)$ according to equation (1). Once this step is complete, the algorithm has a provisional estimate of which sequences belong to which cluster. In the maximization step the algorithm re-computes the transition probabilities $p\left(x_{i} \mid x_{i-1}, c_{k}\right)$ for each cluster $c_{k}$ based on the sequences that belong to that cluster. After the maximization step, the next expectation step will produce different results from the previous iteration, since $p\left(\boldsymbol{x} \mid c_{k}\right)$ will now be computed with the updated values of $p\left(x_{i} \mid x_{i-1}, c_{k}\right)$. The algorithm converges when there is no change in the values of these model parameters.

\section{Experiment \#1: Mining human activity observations}

The first experiment is taken from a research project that aims at discovering recurrent action patterns from action repositories [25]. This experiment was motivated by the difficulties encountered in the manual extraction of action patterns for log sizes of a few hundred actions. Thus, the aim was to test the ability of the sequence clustering algorithm to support manual identification of recurrent action sequences from action logs, where no information of the sequence associated with each individual action was available. Rather than finding Markov chains, the goal here was to evaluate the soundness of the sequence clusters provided by the algorithm.

The experimental data represents the actions of a software development team comprising four software developers and a project leader [24]. The team develops web applications and performs systems analysis, design, programming, test and maintenance activities. The action $\log$ was collected within an observation period of three weeks, during which the team members performed tasks on the following applications: (1) Suppliers, (2) Claims, (3) Customer Correspondence (called Mail application), (4) Evictions and (5) Marketing Campaigns. The team leader performed both system development and project management tasks.

Team observation was carried out by its own members by registering their actions and interactions in chronological order ${ }^{1}$. Both computer- and non-computer-supported actions and interactions were registered, each by means of a summarizing sentence. These sentences were first parsed using grammatical rules to separate the subject and predicate (verb and its complements). Synonym verbs were replaced by a single verb to avoid inconsistencies. Each action and interaction description was augmented with a set of application, information and human resources involved. The results were further

${ }^{1}$ For details on the modeling concepts of action, interaction and context please refer to [21]. 
structured as described in [23] into an event table as shown in figure 3. The data collected over three weeks led to a table with 534 entries.

\begin{tabular}{c|cccccccc}
\hline$\#$ & Day & $\begin{array}{c}\text { Actor } \\
\text { Send. }\end{array}$ & Rec. & $\begin{array}{c}\text { Action. } \\
\text { Interacc. }\end{array}$ & Description & Tools & Information & $\begin{array}{c}\text { Human } \\
\text { competencies }\end{array}$ \\
\hline $\mathbf{8}$ & $6-01$ & Catarina & & SOLVE & $\begin{array}{c}\text { automatic table } \\
\text { update problem }\end{array}$ & $\begin{array}{c}\text { Sql Server, mes- } \\
\text { sage manage- } \\
\text { ment application }\end{array}$ & $\begin{array}{c}\text { Sql Server and mes- } \\
\text { sage management } \\
\text { application documen- } \\
\text { tation }\end{array}$ & $\begin{array}{c}\text { programming \& de- } \\
\text { bugging skills }\end{array}$ \\
\hline 9 & $6-01$ & Catarina & Mariana & PROPOSE & $\begin{array}{c}\text { solution to auto- } \\
\text { matic table up- } \\
\text { date problem }\end{array}$ & & \\
\hline 10 & $6-01$ & Mariana & Catarina & ACCEPT & $\begin{array}{c}\text { solution to auto- } \\
\text { matic table up- } \\
\text { date problem }\end{array}$ & & \\
\hline
\end{tabular}

Fig. 3. Examples of structured actions and interactions collected during observation [22]

By identifying the action contexts of each actor [23] it was possible to group events that belong to the same or to intimately related tasks. This grouping into contexts can be done manually or, in case of large data sets, applying a clustering algorithm can provide a good starting point [22]. For the team leader alone, 12 different action contexts have been identified. Given the chronological order of events within each personal context and the interactions that took place between actors, it was possible to determine the sequences of events that took place across actors. This led to a number of rather long sequences, which were then broken down into shorter, scope-delimited tasks. About 140 tasks were found.

A brief analysis these task sequences revealed two issues. The first was that some of these tasks were not actually sequences, but just arbitrary repetitions of the same action. For example, all team members had at least one task in which they repeated the action "program" from 2 to 20 times. Therefore, consecutive repeating steps within each sequence were eliminated, and sequences ending up with just one single step were discarded. Figure 4 shows the total number of occurrences of each action, both before and after repeating steps were eliminated.

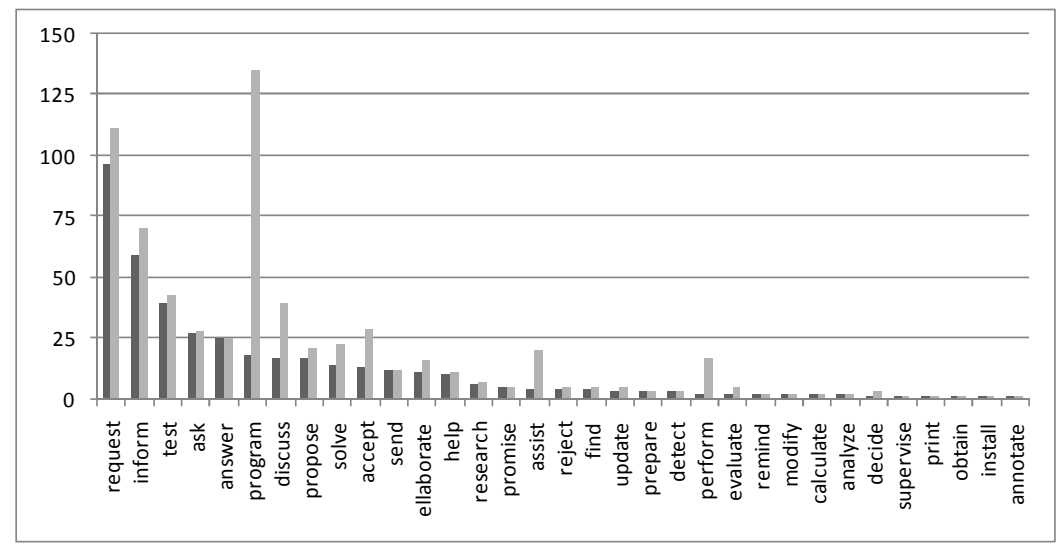

Fig. 4. Total number of occurrences for each action, both before (light column) and after (dark column) eliminating repeating steps, ordered by decreasing number of the latter. 
The second issue was that the relatively high number of different actions led to a set of very dissimilar sequences, despite the fact that most of them shared a limited set of common actions. For example, most tasks involve some form of "request", whereas the action "annotate" happened only once in the entire study. This suggests that the emphasis should be put on highly recurrent actions, which provide the skeleton for most sequences. The least recurrent actions (in the tail of figure 4) represent ad-hoc variations that provide no real insight into the structure of tasks. The last pre-processing stage was therefore to decide on a threshold for the number of occurrences; only actions above that threshold were allowed to remain in the sequences.

Once these pre-processing stages were complete, it was straightforward to build the case and nested tables for the sequence clustering algorithm. In order to present a complete result set, here we will use a relatively high threshold of 20 minimum occurrences. This means that only the first five actions in figure 4 will be allowed. As a consequence, the sequences will also be rather short. Figure 5 shows the results of applying the algorithm to the input sequences. The sequences have been grouped into five clusters.

$\mathrm{c}_{1}$

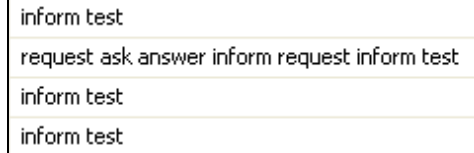

\begin{tabular}{|l|l|}
\hline ask answer \\
\hline$C_{3}$ & ask answer \\
\hline ask answer \\
ask answer \\
ask answer \\
ask answer \\
ask answer \\
ask answer \\
ask answer \\
ask answer \\
ask answer \\
ask answer
\end{tabular}
$\mathrm{C}_{5}$

request inform test inform
request ask answer request inform
request inform test inform
request inform test inform
request inform test inform test

\begin{tabular}{|l|l|}
\hline request test \\
request test \\
request test \\
request test \\
request test \\
request test \\
request inform request test \\
request test \\
request test \\
request test \\
request test \\
request test \\
request test
\end{tabular}

Fig. 5. Results of applying the sequence clustering algorithm to a set of input sequences restricted to five different actions only.

It is arguable whether some sequences should have ended up in a particular cluster. Both cluster $\mathrm{c}_{1}$ and cluster $\mathrm{c}_{4}$ contain one sequence that would make as much sense if it had shown up in another cluster. The key issue here is that similar sequences actually ended up in the same cluster, and that each cluster has its own distinctive features. 
Since the goal was to determine the effectiveness of the algorithm in obtaining meaningful clusters, evaluating the clustering results in this case requires knowledge of the problem domain. In terms of the particular business context, and despite the fact that the set of actions is so limited, it is still possible to draw meaningful conclusions from the results in figure 5 :

- The sequences inform-test (cluster $\mathrm{c}_{1}$ ) and request-test (cluster $\mathrm{c}_{4}$ ) concern software integration tests. Team members confirmed that integration tests are performed either upon explicit request or when the project leader is informed of the result of previous tests. Clusters $c_{1}$ and $c_{4}$ capture these two scenarios. The sequence inform-test actually comprises the states analyze-inform-test, but the action "analyze" was not recorded since it is usually performed by an individual that was not observed in this study.

- The sequences request-inform-test-request (cluster $\mathrm{c}_{2}$ ) and request-inform-testinform (cluster $\mathrm{c}_{5}$ ) concern software publishing activities. These sequences have an additional state - request-publish-inform-test-request and request-publishinform-test-inform - but the action "publish" is also performed by an unobserved member. In all these cases, it is remarkable the algorithm was able to distinguish these activities even though such a key action was missing.

- The sequence ask-answer (cluster $c_{3}$ ) occurs in several kinds of tasks, but mostly in connection with team members helping each other.

\section{Experiment \#2: Mining database system traces}

In the previous experiment, the application of sequence clustering was just the final phase after several weeks of collecting and pre-processing data. In this second experiment, the goal was to devise a scenario in which all these steps would be as automated as possible. Inspired by the bank experiment, we developed an application to perform simple operations over a fictitious banking database. Examples of such operations are: creating a checking account, creating a savings account, creating a loan, paying a loan, etc. Each of these operations comprises several database queries that insert, select, update or delete records in several tables. Operations requiring transactional control were implemented inside stored procedures, so as not to clutter the $\log$.

Creating a checking account for a new customer involves the following steps: (1) create a new customer, (2) create a new account at the branch, (3) save the account as a checking account with a certain withdrawal limit, (4) associate the customer as a depositor of the account, and (5) associate an employee as account manager for that customer. In terms of SQL, this operation would look like:

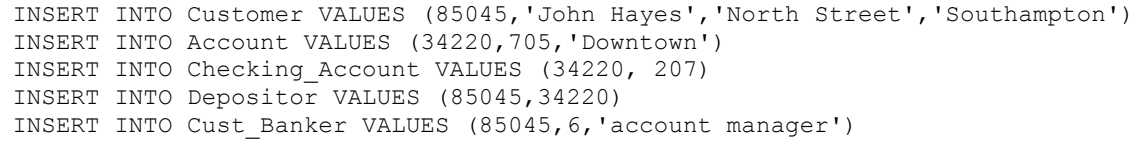

The steps may be performed in this or in a slight different order. In total, there are four variations for this sequence. 
Creating a savings account takes different steps: (1) choose a checking account belonging to the customer, (2) create a new account at the branch, (3) save the account as a savings account with a certain interest rate, (4) associate the customer as a depositor of the account, and (5) transfer the initial funds from the checking account to the newly created savings account. There are two variations for this sequence; the steps in the order just described correspond to the following queries:

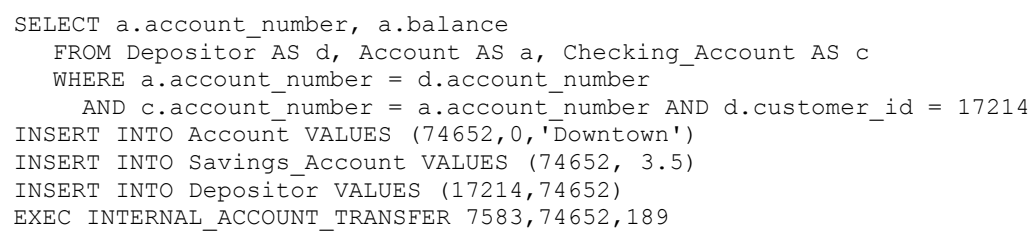

In this experiment, a simulator generates a large amount of these and other operations. The queries from different operations are sent to the database system randomly interleaved, in order to simulate the concurrent execution of both different and similar operations. As the operations are being performed, they are captured as a trace using the SQL Server Profiler, a tool for monitoring the SQL Server Database Engine and capturing data about each event. Figure 6 illustrates how the data is captured with the Profiler. There is no case id or any other information that explicitly indicates that an event belongs to a certain sequence. As it stands, the trace is just an unclassified stream of events.

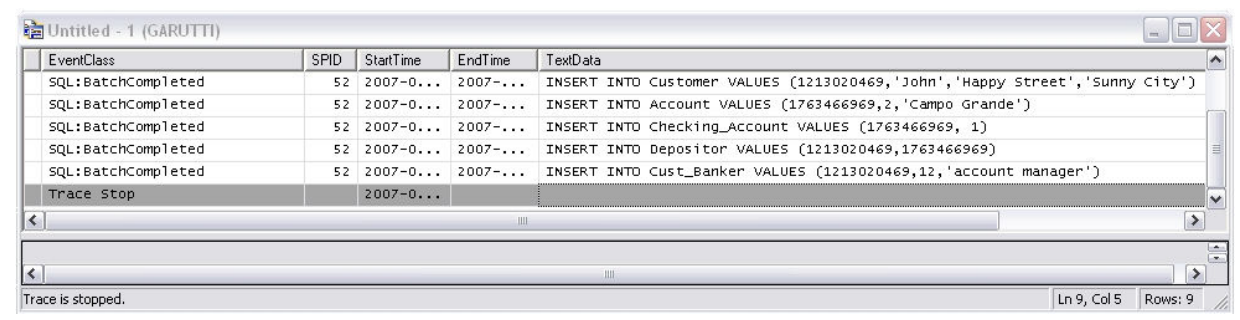

Fig. 6. A database system trace as captured by the SQL Server Profiler. The data can be saved to a file or to a database table as it is being captured.

The second stage of this experiment is supported by the SequenceBuilder module, a software component that pre-processes the trace in order to create the case and nested tables for sequence clustering. However, the algorithm requires a set of independent sequences grouped by a case id and sorted by a sequential number (as shown earlier in figure 1). This means that SequenceBuilder must figure out where each sequence begins and ends, and find the events that belong to the sequence. The database trace contains profile information - such as date, username, client application, connection identifier, etc. - that could provide an indication of whether two events are related or not. But this information is not enough to find an accurate set of sequences. In [15] the authors make use of similar event logs, but the case id is given for each event. For the $\log$ shown in figure 6 some kind of reliable method for sequence identification had to be found. 
The chosen method was to analyze the content of each query in order to determine whether it used the same objects of other queries or not. By retrieving the parameter values of each query, two queries that are close together in the log and use the same customer id, for example, are very likely to belong to the same sequence. And even if they do not share the same parameters, but a third query uses both of their parameter values, then all the three queries are likely to belong to the same sequence. This led to the idea of computing the graph of relations between queries in the trace: events connected directly or indirectly through other nodes belong to the same sequence; the islands in the graph correspond to different sequences. Figure 7 shows the connecting graphs for the queries described earlier.

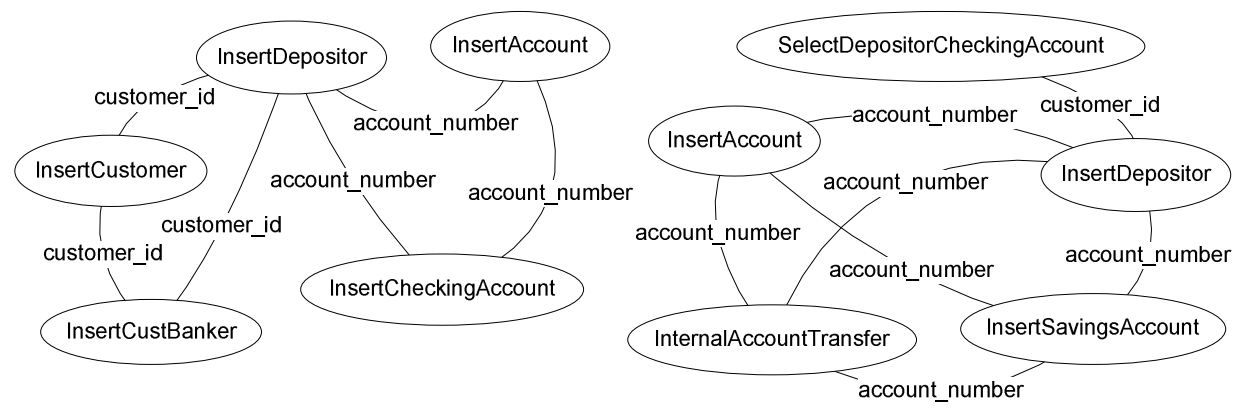

Fig. 7. Links between the queries in the bank operations described earlier. Each link has the name of the parameter whose value is equal in the two queries. Separate sub-graphs correspond to different sequences.

The whole graph can be computed at most in $O\left(N^{2}\right)$ by comparing every pair of events in the log. The graph is saved into table form in the database, where each node may have many connections to other nodes. A recursive query then retrieves the set of nodes in each sub-graph, until there are no more nodes to retrieve. As the nodes are being retrieved, they are sorted by the chronological order in which they originally appeared in the trace. The incoming nodes are assigned a sequential number and saved to a nested table, with a different case id for each sequence. The case table is then generated by retrieving the set of all distinct case ids.

This simple method works well in all cases except one: when the same object - be it the customer, account, loan, etc. - shows up in another instance of the same or different sequence. This may happen because the same customer opens more than one savings account, because the customer sends payment for a previously created loan, etc. The problem is illustrated in figure 8 , where there should be three sequences but there are only two since a link is established to a later sequence that refers to the same object. If these long, unintended sequences are left in the input data, they will ruin the sequence clustering results since the algorithm will try to find some way to fit these sequences in by generating Markov chains that are able to produce them. 


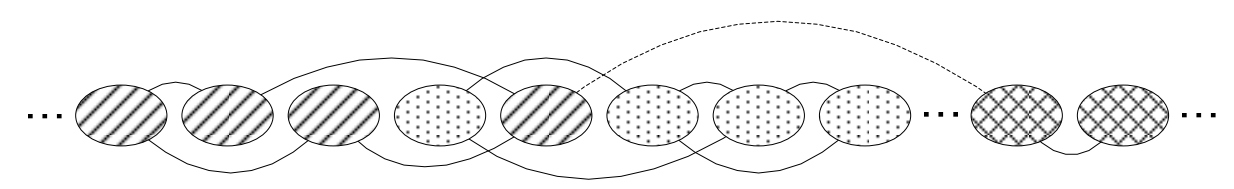

Fig. 8. When running through the log, links may be established between events that actually belong to different sequences.

Fortunately, this phenomenon can be detected using a simple heuristic based on the average length of links between events. A link whose length is noticeably higher than the average length of all links is likely to be a spurious connection rather than a meaningful relationship between two events. Suppose, for example, that links with length over two times the average are rejected. In the example shown in figure 8 , the average length is $(18+x) / 11$ where $x$ is the length of the dashed link. We reject the dashed link if $x \geq 2 *(18+x) / 11$ which gives $x \geq 4$ which is obviously appropriate in this example, where the maximum length of "true" links is 3 . Of course, these decisions are all but trivial, since the "false" links could actually provide insight into higher-level patterns of behaviour, although this possibility in not being pursued at the time of writing.

Figure 9 shows five of the eight clusters found for a database trace with about 100 sequences. The first three clusters $-c_{2}, c_{4}$ and $c_{7}-$ are three of the four variations of creating a checking account; clusters $\mathrm{c}_{5}$ and $\mathrm{c}_{6}$ represent the two variations of creating a savings account. The remaining clusters had similar results for other kinds of operations dealing with loans. The algorithm was able to clearly distinguish all operations and their variations, and put each sequence in a separate cluster. As a result, the Markov chains turned into deterministic graphs, since all transitions probabilities equal $100 \%$.

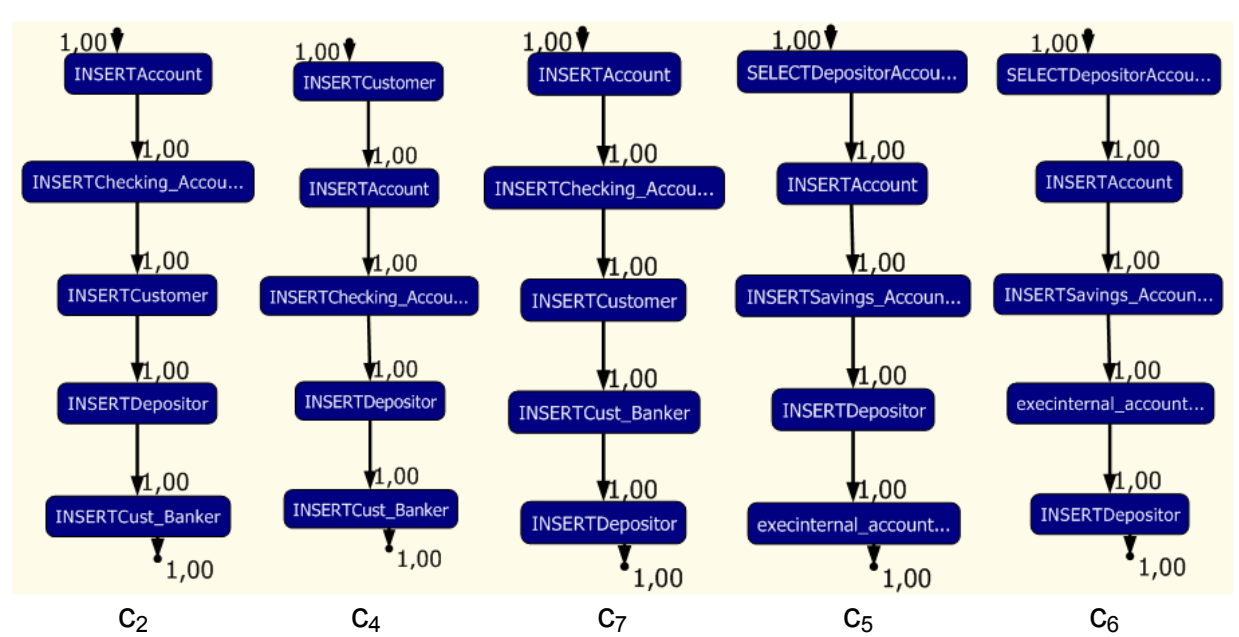

Fig. 9. Markov chains associated with five of the eight clusters found in the bank example. 
In terms of similarity between the sequences, the algorithm was able to find that clusters $\mathrm{c}_{2}, \mathrm{c}_{4}$, and $\mathrm{c}_{7}$ are very similar, and the same happens with clusters $\mathrm{c}_{5}$ and $\mathrm{c}_{6}$. Figure 10 shows the cluster diagram for the same results, where the shading of lines that connect two clusters represents the strength of the similarity between those clusters, and the shade of each cluster represents its population. From the diagram it becomes apparent that there is a cluster $c_{8}$ which is similar to clusters $c_{2}, c_{4}$, and $c_{7}$. Indeed, cluster $c_{8}$ contains the fourth variation of creating a checking account. It corresponds to the steps of cluster $\mathrm{c}_{4}$ being executed in the order $(1) \rightarrow(5) \rightarrow(2) \rightarrow(3)$ $\rightarrow(4)$.

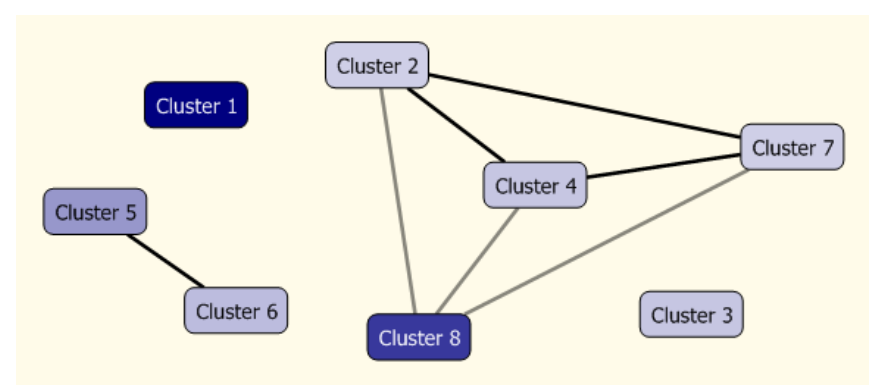

Fig. 10. Cluster diagram for the results obtained in the bank example.

\section{Conclusion}

Sequence clustering is a powerful technique to sort out different behaviours and to provide insight into the underlying structure of those behaviours. This insight is especially useful when approaching new scenarios, that the business process analyst may not be familiar with, or where the potential for process mining is yet uncertain. It can actually become a valuable tool as a first approach to process mining, when the event $\log$ is too large to be manually handled and the presence of ad-hoc behaviour makes it impossible for automated processing by more deterministic algorithms.

However, in order to obtain meaningful results via sequence clustering, the input data must be carefully prepared and pre-processed. The experiments described in this paper show that the challenge is actually in identifying and compiling the set of input sequences, rather than applying the algorithm, which is straightforward. In experiment \#1 the sequences were delimited manually and then streamlined by discarding infrequent actions. In experiment \#2 the sequences were delimited automatically by means of a criterion that allowed links to be established between events. In both cases, the case id was assigned based on application-specific heuristics.

These experiments confirm the ability of sequence clustering to identify different tasks and to discover their composition in terms of elemental steps. In future work, further sequence analysis over these clusters is expected to provide insight into behaviour at the process level. 


\section{References}

1. W. van der Aalst, "Workflow mining: A survey of issues and approaches", Data \& Knowledge Engineering, 47, pp. 237-267, 2003

2. W. van der Aalst, A. Medeiros, A. Weijters, "Genetic Process Mining", Applications and Theory of Petri Nets 2005, LNCS 3536, pp.48-69, Springer, 2005

3. W. van der Aalst, A. Weijters, "Process Mining: A Research Agenda", Computers in Industry, 53(3):231-244, 2004

4. W. van der Aalst, T. Weijters, L. Maruster, "Workflow Mining: Discovering Process Models from Event Logs", IEEE Transactions on Knowledge and Data Engineering, 16(9):1128-1142, September 2004

5. R. Agrawal, D. Gunopulos, F. Leymann, "Mining Process Models from Workflow Logs", Proceedings of the 6th International Conference on Extending Database Technology: Advances in Database Technology, LNCS 1377, pp.469-483, Springer, 1998

6. I. Cadez, D. Heckerman, C. Meek, P. Smyth, S. White, "Model-Based Clustering and Visualization of Navigation Patterns on a Web Site", Data Mining and Knowledge Discovery, 7(4): 399-424, October 2003

7. Y. Chen, K. Reilly, A. Sprague, Z. Guan, "SEQOPTICS: a protein sequence clustering system", BMC Bioinformatics, 7(Suppl 4):S10, 2006

8. J. Cook, A. Wolf, "Automating process discovery through event-data analysis", Proceedings of the 17th International Conference on Software Engineering, pp.7382, ACM Press, 1995

9. A. Dempster, N. Laird, D. Rubin, "Maximum Likelihood from Incomplete Data via the EM Algorithm", Journal of the Royal Statistical Society, Series B, 39(1):138,1977

10. B. van Dongen, W. van der Aalst, "Multi-Phase Process Mining: Building Instance Graphs", International Conference on Conceptual Modeling, LNCS 3288, pp.362376, Springer, 2004

11. B. van Dongen, W. van der Aalst, "Multi-Phase Mining: Aggregating Instances Graphs into EPCs and Petri Nets", Proceedings of the Second International Workshop on Applications of Petri Nets to Coordination, Workflow and Business Process Management, pp.35-58, 2005

12. A. Enright, S. van Dongen, C. Ouzounis, "An efficient algorithm for large-scale detection of protein families", Nucleic Acids Research, 30(7):1575-1584, 2002

13. A. Enright, C. Ouzounis "GeneRAGE: a robust algorithm for sequence clustering and domain detection”, Bioinformatics, 16(5): 451-457, 2000

14. G. Greco, A. Guzzo, L. Pontieri, "Mining Hierarchies of Models: From Abstract Views to Concrete Specifications", Proceedings of the 3rd International Conference on Business Process Management, BPM 2005, pp. 32-47, 2005

15. C. Günther, W. van der Aalst, "Mining Activity Clusters from Low-Level Event Logs", BETA Working Paper Series, WP 165, Eindhoven University of Technology, Eindhoven, 2006

16. J. Herbst, D. Karagiannis, "Integrating Machine Learning and Workflow Management to Support Acquisition and Adaptation of Workflow Models", Proceedings of the 9th International Workshop on Database and Expert Systems Applications, pp.745-752, 1998 
17. W. Li, L. Jaroszewski, A. Godzik, "Sequence clustering strategies improve remote homology recognitions while reducing search times", Protein Engineering, 15(8): 643-649, August 2002

18. Microsoft Corporation, "OLE DB for Data Mining Specification", Version 1.0, July 2000

19. Z. Tang, J. MacLennan, "Data Mining with SQL Server 2005”, Wiley, 2005

20. T. Wu, C. Watanabe, "GMAP: a genomic mapping and alignment program for mRNA and EST sequences", Bioinformatics, 21(9):1859-1875, 2005

21. M. Zacarias, A. Caetano, H. Pinto, J. Tribolet, "Modeling Contexts for Business Process Oriented Knowledge Support", Workshop on Knowledge Management for Distributed Agile Processes: Models, Techniques, and Infrastructure, April 2005

22. M. Zacarias, R. Gomes, J. Coimbra, H. Pinto, J. Tribolet, "Discovering Personal Action Contexts with SQL Server Integration and Analysis Services", Proceedings of the 2nd International Conference on Innovative Views of .NET Technologies, October 2006

23. M. Zacarias, A. Marques, H. Pinto, J. Tribolet, "Enhancing Collaboration Services with Business Context Models", International Workshop on Cooperative Systems and Context, 5th International and Interdisciplinary Conference on Modeling and Using Context, July 2005

24. M. Zacarias, H. Pinto, J. Tribolet, "A Context-based Approach to Discover Multitasking Behavior at Work", Proceedings of the 5th International Workshop on Task Models and Diagrams for User Interface Design, October 2006

25. M. Zacarias, H. Pinto, J. Tribolet, "Reverse-engineering of Personal and Interpersonal Work Practices: A Context-Based Approach", International Workshop on Role of Contextualization in Human Tasks: Enriching Actors (CHUT-07) held in conjunction with CONTEXT 07, Denmark, August 2007 